\title{
HIPERTENSÃO ARTERIAL EM PROFISSIONAIS QUE ATUAM EM SERVIÇOS DE ATENDIMENTO PRÉ-HOSPITALAR
}

\author{
Luciane Cesira Cavagioni ${ }^{1}$, Angela Maria Geraldo Pierin ${ }^{2}$
}

\footnotetext{
${ }^{1}$ Doutoranda do Programa de Enfermagem na Saúde do Adulto da Escola de Enfermagem da Universidade de São Paulo (EEUSP). São Paulo, Brasil. E-mail: cavagioni@usp.br

${ }^{2}$ Livre-docente. Professora Titular do Departamento de Enfermagem Médico-Cirúrgica da EEUSP. São Paulo, Brasil. E-mail: pierin@usp.br
}

RESUMO: Avaliou-se a prevalência de hipertensão e variáveis relacionadas em 154 profissionais de serviço pré-hospitalar pela medida casual da pressão e monitorização ambulatorial da pressão. A prevalência de hipertensão na medida casual foi 33,1\% e 26,6\% na monitorização ambulatorial da pressão arterial. A presença de hipertensão se associou (odds ratio=OR): a) medida casual da pressão, com o HDL-c $>40 \mathrm{mg} / \mathrm{dL}$ (OR=0,25); b) monitorização ambulatorial (24 h) com glicemia > $110 \mathrm{mg} / \mathrm{dL}$ (OR:9,98), sexo masculino $(\mathrm{OR}=2,71)$, trabalhar cansado raramente/nunca $(\mathrm{OR}=0,19)$ e às vezes $(\mathrm{OR}=0,17) ; \mathbf{c})$ monitorização ambulatorial (vigília) com glicemia $>110 \mathrm{mg} / \mathrm{dL}(\mathrm{OR}=11,18)$, sexo masculino $(\mathrm{OR}=3,24)$, trabalhar cansado raramente/nunca $(\mathrm{OR}=0,14)$ e às vezes $(\mathrm{OR}=0,16)$, índice de massa corporal $(\mathrm{OR}=1,10)$, trabalhar cansado raramente/ nunca $(\mathrm{OR}=0,14)$ e às vezes $(\mathrm{OR}=0,16)$; e d) monitorização ambulatorial (sono) com sono diurno $(\mathrm{OR}=0,14)$ e índice de massa corporal $(\mathrm{OR}=1,11)$. Foi elevada a prevalência de hipertensão e fatores de risco associados. DESCRITORES: Hipertensão arterial. Atendimento pré-hospitalar. Monitorização ambulatorial da pressão arterial. Saúde do trabalhador.

\section{HYPERTENSION IN PROFESSIONALS WORKING IN PREHOSPITAL CARE SERVICES}

\begin{abstract}
This study evaluated the prevalence of hypertension and related variables among 154 pre-hospital care service professionals, measuring for causal blood pressure and ambulatory blood pressure. The prevalence of hypertension by causal blood pressure measurement was $33.1 \%$ and ambulatory blood pressure was $26.6 \%$. Hypertension was associated with (odds ratio=OR): a) causal blood pressure measurement, with HDL-c $>40 \mathrm{mg} / \mathrm{dL}(\mathrm{OR}=0.25)$; b) 24-hour ambulatory blood pressure with blood glucose levels $>110 \mathrm{mg} / \mathrm{dL}(\mathrm{OR}=9.98)$, male gender $(\mathrm{OR}=2.71)$, rarely / never working tired $(\mathrm{OR}=0.19)$, and sometimes working tired $(\mathrm{OR}=0.17)$; c) daytime ambulatory blood pressure with glucose $>110 \mathrm{mg} / \mathrm{dL}(\mathrm{OR}=11.18)$, male gender $(\mathrm{OR}=3.24)$, rarely/never working tired $(\mathrm{OR}=0.14)$, sometimes working tired $(\mathrm{OR}=0.16)$, body mass index $(\mathrm{OR}=1.10)$, rarely/never working tired $(\mathrm{OR}=0.14)$, and sometimes working tired $(\mathrm{OR}=0.16)$; and $\mathrm{d})$ ambulatory blood pressure during sleep with daytime sleep $(\mathrm{OR}=0.14)$ and body mass index $(\mathrm{OR}=1.11)$. Hypertension prevalence was high and was associated with changeable cardiovascular risk factors.
\end{abstract}

DESCRIPTORS: Arterial hypertension. Prehospital care. Ambulatory blood pressure monitoring. Occupational health.

\section{HIPERTENSIÓN ARTERIAL EN PROFESIONALES QUE TRABAJAN EN SERVICIOS DE ATENCIÓN PREHOSPITALARIA}

\begin{abstract}
RESUMEN: Se evaluó la prevalencia de hipertensión arterial y las variables relacionadas en 154 profesionales que trabajan en servicios de atención prehospitalaria. Se efectuaron mediciones casuales y por monitorización ambulatoria de presión arterial. La prevalencia de hipertensión por medición casual fue del 33,0\%, y del 26,6\% en las mediciones casuales y por monitorización ambulatoria de presión arterial. La presencia de hipertensión se asoció (odds ratio=OR): a) medición casual, con el HDL-c $>40$ mg/dL (OR=0,25); b) mediciones casuales y por monitorización ambulatoria de presión arteria ( 24 hs.) con glucemia $>110 \mathrm{mg} / \mathrm{dL}(\mathrm{OR}=9,98)$, hombres $(\mathrm{OR}=2,71)$, trabajar cansado raramente/ nunca $(\mathrm{OR}=0,19)$, y a veces $(\mathrm{OR}=0,17)$; $)$ mediciones casuales y por monitorización ambulatoria de presión arterial (vigilia) con glucemia $>110 \mathrm{mg} / \mathrm{dL}(\mathrm{OR}=11,18)$, hombres $(\mathrm{OR}=3,24)$, trabajar cansado raramente/nunca $(\mathrm{OR}=0,14)$, a veces $(\mathrm{OR}=0,16)$, índice de masa corporal $(\mathrm{OR}=1,10)$, trabajar cansado raramente/nunca $(\mathrm{OR}=0,14)$, a veces $(\mathrm{OR}=0,016)$; $\mathrm{y}$, $\mathrm{d})$ mediciones casuales y por monitorización ambulatoria de presión arterial (sueño) con sueño diurno $(\mathrm{OR}=0,14)$ e índice de masa corporal $(\mathrm{OR}=1,11)$. Se encontró una alta prevalencia de hipertensión arterial asociada a factores de riesgo cardiovascular.
\end{abstract}

DESCRIPTORES: Hipertensión arterial. Atención prehospitalaria. Monitorización ambulatoria de la presión arterial. Salud laboral. 


\section{INTRODUÇÃO}

O Brasil tem experimentado, nas últimas décadas, importantes transformações no seu padrão de morbi-mortalidade, em função dos processos de transição epidemiológica, demográfica e nutricional. Houve uma importante redução da mortalidade por doenças infecciosas e parasitárias, mas, por outro lado, ocorreu aumento em mais de três vezes das doenças cardiovasculares. A proporção de brasileiros diagnosticados com hipertensão arterial cresceu de 21,5\%, em 2006, para 24,4\%, em 2009. ${ }^{1}$

Dentre as doenças do aparelho circulatório destaca-se a hipertensão arterial, que tem como principal agravante o fato de ser, na maioria dos casos, assintomática. O desenvolvimento da hipertensão arterial depende da interação entre os fatores genéticos, neuro-humorais, comportamentais e ambientais. Porém, ainda não é bem conhecida como se processa essa interação. A única maneira de se identificar a hipertensão arterial é realizando a medida da pressão. A medida casual da pressão arterial, geralmente realizada em consultório, constitui o método tradicional do diagnóstico da hipertensão arterial. Entretanto, evidências demonstraram que essa medida pode não refletir a real pressão arterial das pessoas. A influência de fatores como o ambiente, onde se realiza a medida, e o observador, quem executa a medida, podem ocasionar o chamado fenômeno do avental branco. ${ }^{2-3}$ Uma estratégia utilizada para eliminar a possível discrepância da medida casual da pressão arterial é a Monitorização Ambulatorial da Pressão Arterial (MAPA). Com o advento da MAPA surge um novo capítulo na história da esfigmomanometria, com vantagens, entre outras, de atenuar o efeito do observador e do ambiente sobre a pressão arterial, avaliar a pressão durante o sono e a resposta terapêutica durante um período de 24 horas. $^{4}$

Existem em nosso meio, alguns estudos em categorias profissionais específicas para avaliar a prevalência de hipertensão na área da saúde. Estudo realizado na cidade de Salvador, Bahia, com trabalhadoras da área de enfermagem de um hospital mostrou prevalência de $36 \% .{ }^{5}$ Em funcionários de um complexo hospitalar da cidade de São Paulo, a prevalência foi de $26 \%{ }^{6}$ Destaca-se que em todos esses estudos a avaliação da pressão arterial foi feita exclusivamente pela medida casual. Porém, estudos com população de trabalhadores específicos como bombeiros e médicos usaram a MAPA para avaliar a pressão arterial e os resultados mostraram elevações significativas da pressão arterial durante o período de trabalho. ${ }^{7,8}$ A elevação da pressão arterial pode acarretar aumento do risco cardiovascular entre esses profissionais. A exposição crônica de indivíduos suscetíveis a condições de trabalho estressantes, pode ser responsabilizada por aumentos pressóricos persistentes e significativos, conduzindo ao quadro hipertensivo.

Dessa forma, os profissionais de saúde que atuam em serviços de atendimento médico pré-hospitalar estariam expostos a ambientes conflituosos, alta exigência de trabalho, estresse físico e mental, alteração no ritmo circadiano, dentre outros fatores que podem contribuir para o surgimento da hipertensão arterial e consequentemente aumentar o risco cardiovascular. A partir do exposto, o presente estudo teve por objetivos: conhecer a prevalência de hipertensão arterial nesses profissionais com o uso da medida casual da pressão arterial e MAPA e relacionar com variáveis sócio, demográficas e comportamentais.

\section{MATERIAL E MÉTODO}

Trata-se de um estudo transversal, com amostra aleatória em 154 profissionais da área da saúde, que atuavam nos seguintes serviços de atendimento pré-hospitalar: Serviço de Atendimento Móvel de Urgência (SAMU) 192 e Grupo de Atendimento às Urgências (GRAU) 193, ambos da cidade de São Paulo, com abrangência nas quatro regiões da cidade (Norte, Sul, Leste e Oeste) e SAMU-Vale do Ribeira-SP, localizada na Rodovia BR-116. Após a obtenção dos números das bases operacionais, localizadas nas quatro regiões de São Paulo e ao longo da BR-116, os locais para a coleta dos dados foram sorteados e todos os profissionais desses locais foram convidados, através de contato telefônico, e-mail ou pessoalmente a participarem do estudo. Esse processo se repetiu até alcançar o número calculado da amostra. O cálculo do tamanho da amostra foi realizado para uma variável dicotômica em estudo descritivo onde a proporção de casos esperados foi de $20 \%$, com a intervalo de confiança de 0,10 (0,5 acima e 0,5 abaixo) a um nível de confiança de $99 \%$ obtendo-se 130 profissionais. Os critérios de inclusão foram: ser profissional da área da saúde, atuar diretamente no atendimento às vítimas e ter no mínimo três meses de atividade profissional no serviço. Todos profissionais assinaram o termo de consentimento e o projeto foi aprovado pelo Comitê de Ética da Escola de Enfermagem da Universidade de São Paulo, sob n ${ }^{0} 742 / 2008$. A coleta dos dados iniciou em agosto de 2008, com término em janeiro de 2009. Antes do início do plantão dos profissionais, em seu respectivo serviço 
de atendimento pré-hospitalar, a pesquisadora realizou a medida casual da pressão arterial. Foram efetuadas três medidas, com intervalo de um a dois minutos, com aparelho automático validado (OMROM HEM 705CP) seguindo as orientações das V Diretrizes Brasileiras de Hipertensão: ${ }^{2}$ na posição sentada após dois minutos de repouso; com as costas apoiadas e pernas descruzadas; manguito com largura e comprimento adequados à circunferência do braço; estar com a bexiga urinária vazia; e não ter fumado, ingerido bebidas alcoólicas, café e alimentos a menos de 30 minutos. Para a análise estatística foi considerada a média das duas últimas aferições e os níveis pressóricos dos participantes classificados em hipertensos para pressão sistólica $\geq 140 \mathrm{mmHg}$ e/ou pressão diastólica $\geq 90 \mathrm{mmHg}$ e/ ou a referência de uso de anti-hipertensivos.

Após a verificação da pressão casual, foi instalado o monitor de medida da pressão ambulatorial. Usou-se aparelho tipo oscilométrico validado (Spacelabs 90217 e 90207) seguiu-se o protocolo das IV Diretrizes para o uso da MAPA: ${ }^{4}$ manguito de tamanho adequado à circunferência do braço; posicionado 2 a $3 \mathrm{~cm}$ acima da fossa cubital no braço não dominante; o participante foi orientado para permanecer com o braço ao longo do corpo e sem movimentá-lo quando as medidas fossem realizadas automaticamente. Os critérios aceitos como válidos para análise da MAPA foram: duração mínima de $24 \mathrm{~h}$, com pelo menos $80 \%$ de leituras válidas do total de medidas; e ausência de leitura superior ou igual a duas horas. Os critérios de normalidade da MAPA foram (sistólica e diastólica, respectivamente): média de 24 horas, $\leq 130 \mathrm{mmHg}$ / $\leq 80 \mathrm{mmHg}$; média da vigília, $\leq 135 \mathrm{mmHg}$ / $\leq 85$ $\mathrm{mmHg}$; e média do sono, $\leq 120 \mathrm{mmHg} / \leq 70 \mathrm{mmHg}$. Para a determinação do período de sono, foi considerado o horário relatado no diário de atividades.

Os participantes responderam um questionário que incluía questões relativas às seguintes variáveis: sexo, idade (em anos), categoria profissional (médicos, enfermeiros ou auxiliares de enfermagem), tempo de formado, carga horária semanal, frequência de trabalho, tabagismo (sim para qualquer quantidade de cigarro diária e os que pararam há menos de 1 ano), ingestão de bebidas alcoólicas (bebedores: os que faziam uso de bebida alcoólica, independentemente do tipo e quantidade), atividade física regular (pelo menos 30 minutos de atividade física de forma continua ou acumulada, três ou mais vezes por semana), referência pessoal e familiar de hipertensão arterial e se dormiu durante o dia quando estava de plantão no serviço de atendimento pré-hospitalar.
Realizou-se a determinação do peso (em kilogramas) e a altura (em metros), através de uma balança antropométrica mecânica, para cálculo do Índice de Massa Corpórea (IMC: peso/altura²). Os profissionais foram classificados pelos critérios da Organização Mundial da Saúde em: IMC saudável $\left(<25 \mathrm{~kg} / \mathrm{m}^{2}\right)$, sobrepeso $\left(25\right.$ a $\left.29,9 \mathrm{~kg} / \mathrm{m}^{2}\right)$ e obeso $\left(\geq 30 \mathrm{~kg} / \mathrm{m}^{2}\right) .{ }^{9}$

A circunferência abdominal foi medida no ponto médio entre a crista ilíaca e o rebordo costal, usando-se fita métrica inelástica ajustada ao corpo e classificada de acordo com os critérios da Organização Mundial da Saúde: para o sexo masculino, normal $<94 \mathrm{~cm}$, risco aumentado $\geq 94$ $\mathrm{cm}$ e risco muito aumentado $\geq 102 \mathrm{~cm}$; para o sexo feminino, normal $<80 \mathrm{~cm}$; risco aumentado $\geq 80 \mathrm{~cm}$ e risco muito aumentado $\geq 88 \mathrm{~cm} .{ }^{9}$

A coleta de sangue para realização dos exames laboratoriais foi feita com sistema vacutainer sendo colhido sangue venoso antecubital, após 12 horas de jejum e encaminhado ao laboratório logo em seguida, para dosagem do perfil lipídico e da glicemia. Os participantes foram classificados segundo os critérios da III Diretrizes Brasileiras de Dislipidemias e Diretriz de Prevenção da Aterosclerose $^{10}$ e do Consenso Brasileiro de Diabetes ${ }^{11} \mathrm{e}$ proteína $C$ reativa, em quartis.

Para a análise estatística foi usado o software "Statistical Package for Social Sciences" (SPSS) versão 7.5. As variáveis classificatórias descritivas são apresentadas em frequências absolutas (n) e relativas (\%) e os dados contínuos em média e desvio padrão. As variáveis foram consideradas estatisticamente significativas para $p<0,05$ e analisadas pelo teste qui-quadrado ou teste exato de Fischer quando pertinente. As variáveis que apresentaram significância estatística na análise univariável compuseram o modelo de regressão logística (método forward step wise) para a hipertensão arterial pela medida da pressão casual e pela MAPA.

\section{RESULTADOS}

Foram estudados 154 profissionais, sendo mais da metade composta de enfermeiros (59\%), seguida pelos médicos $(26 \%)$ e auxiliares de enfermagem $(15 \%)$. Houve predomínio do sexo feminino $(60,4 \%)$ e de adultos jovens $(40,9 \pm 7,8$ anos). Foi elevada a referência de antecedentes familiares para hipertensão $(82,5 \%)$, porém, apenas $20,1 \%$ referiram antecedentes pessoais. Em relação aos hábitos de vida, um quinto informou ser tabagista, porém a ingestão de bebidas alcoólicas foi bem mais elevada (47,5\%), o mesmo ocorren- 
do com a falta de atividade física regular (64\%). No tocante às características profissionais, pouco menos da metade $(43,5 \%)$ trabalhava mais de 60 horas semanais e montante bem próximo (45\%) referiu dormir durante o dia quando estava no serviço de atendimento pré-hospitalar. A maioria possuía duas décadas de formação profissional (74\%), trabalhava há 10 anos no serviço $(70 \%)$ e mais da metade referiu trabalhar frequentemente ou algumas vezes cansada $(58,8 \%)$ (Tabela 1$)$.

Tabela 1 - Distribuição das características sócio-demográficas e hábitos de vida dos profissionais que atuam no atendimento pré-hospitalar. São Paulo-SP, 2010

\begin{tabular}{|c|c|c|}
\hline Características & $\mathbf{n}$ & $\%$ \\
\hline \multicolumn{3}{|l|}{ Sexo } \\
\hline Masculino & 61 & 39,6 \\
\hline Feminino & 93 & 60,4 \\
\hline \multicolumn{3}{|l|}{ Idade (anos) } \\
\hline$<40$ & 76 & 49,3 \\
\hline$\geq 40$ & 78 & 50,7 \\
\hline \multicolumn{3}{|l|}{ Média \pm desvio padrão: $40,9 \pm 7,8$} \\
\hline \multicolumn{3}{|l|}{ Anos de formado } \\
\hline $01-20$ & 114 & 74,0 \\
\hline$>20$ & 40 & 26,0 \\
\hline \multicolumn{3}{|l|}{ Categoria profissional } \\
\hline Enfermeiros & 90 & 59,0 \\
\hline Médicos & 41 & 26,0 \\
\hline Auxiliares de enfermagem & 23 & 15,0 \\
\hline \multicolumn{3}{|l|}{ Anos de trabalho na unidade pré-hospitalar } \\
\hline $01 \mid-10$ & 108 & 70,0 \\
\hline $10-\mid 15$ & 46 & 30,0 \\
\hline \multicolumn{3}{|l|}{ Carga de trabalho semanal } \\
\hline $12 \mathrm{l}-\mathrm{l} 30 \mathrm{~h}$ & 21 & 13,6 \\
\hline $30-150 \mathrm{~h}$ & 32 & 20,7 \\
\hline $50-160 \mathrm{~h}$ & 34 & 22,2 \\
\hline$>60 \mathrm{~h}$ & 67 & 43,5 \\
\hline \multicolumn{3}{|l|}{ Trabalha cansado no pré-hospitalar } \\
\hline Algumas vezes & 73 & 47,7 \\
\hline Raramente/nunca & 64 & 41,2 \\
\hline Frequentemente & 17 & 11,1 \\
\hline Sono diurno durante o plantão no pré-hospitalar & 69 & 45,0 \\
\hline Fumo & 31 & 20,1 \\
\hline Ingestão de bebidas alcoólicas & 73 & 47,5 \\
\hline Atividade física & 56 & 35,9 \\
\hline Antecedentes pessoais $p /$ hipertensão arterial & 31 & 20,1 \\
\hline Antecedentes familiares $\mathrm{p} /$ hipertensão arterial & 127 & 82,5 \\
\hline
\end{tabular}

$\mathrm{Na}$ avaliação dos valores da pressão arterial, verificou-se que, pela medida casual, pouco mais de um terço dos participantes $(33,1 \%)$ apresentou níveis compatíveis com hipertensão arterial. Entretanto, considerando-se a MAPA, essa prevalência se reduz levando-se em conta a média do período de $24 \mathrm{~h}(29,3 \%)$ e do período de vigília $(26,6 \%)$. Porém, houve tendência inversa no período de sono, pois mais da metade $(63,0 \%)$ apresentou níveis pressóricos compatíveis com valores de hipertensão arterial. Em relação ao índice de massa corporal $(65,6 \%)$ e a circunferência abdominal $(70,2 \%)$ foram expressivos os percen- tuais dos profissionais que apresentaram índices acima dos considerados saudáveis. A avaliação do perfil lipídico mostrou que pouco mais de um terço $(35,7 \%)$ apresentou níveis de colesterol total na faixa considerada inadequada e LDL-c $(33,8 \%)$ compatível com nível ótimo, enquanto que para a fração HDL-c, mais de um quarto dos profissionais estava na faixa considerada inadequada $(27,9 \%)$. Quanto ao nível de triglicérides, praticamente $30 \%$ estavam na fixa limítrofe ou alterado. Por outro lado, verificou-se baixo índice de glicemia de jejum alterado $(2,6 \%)$ e proteína $C$ reativa no maior quartil $(15,6 \%)$ (Tabela 2$)$. 
Tabela 2 - Características e distribuição da pressão arterial casual e MAPA dos profissionais que atuam no atendimento pré-hospitalar. São Paulo-SP, 2010

\begin{tabular}{|c|c|c|c|}
\hline Variável & $\mathbf{n}$ & $\%$ & Média \pm DP \\
\hline \multicolumn{4}{|l|}{ Pressão arterial casual } \\
\hline$<140 /<90 \mathrm{mmHg}$ & 103 & 66,9 & \\
\hline$\geq 140 / \geq 90 \mathrm{mmHg}$ & 51 & 33,1 & \\
\hline Pressão arterial sistólica $(\mathrm{mmHg})$ & & & $125,3 \pm 14,9$ \\
\hline Pressão arterial diastólica (mmHg) & & & $79,3 \pm 10,8$ \\
\hline \multicolumn{4}{|l|}{ MAPA: Período de 24 horas } \\
\hline$\leq 130 / 80 \mathrm{mmHg}$ & 109 & 70,7 & \\
\hline$>130 / 80 \mathrm{mmHg}$ & 45 & 29,3 & \\
\hline Pressão arterial sistólica & & & $119,1 \pm 10,8$ \\
\hline Pressão arterial diastólica & & & $74,7 \pm 8,0$ \\
\hline \multicolumn{4}{|l|}{ Período de vigília } \\
\hline$\leq 135 / 85 \mathrm{mmHg}$ & 113 & 73,4 & \\
\hline$>135 / 85 \mathrm{mmHg}$ & 41 & 26,6 & \\
\hline Pressão arterial sistólica & & & $122,0 \pm 11,2$ \\
\hline Pressão arterial diastólica & & & $77,5 \pm 8,3$ \\
\hline \multicolumn{4}{|l|}{ Período de sono } \\
\hline$\leq 120 / 70 \mathrm{mmHg}$ & 57 & 37,0 & \\
\hline$>120 / 70 \mathrm{mmHg}$ & 97 & 63,0 & \\
\hline Pressão arterial sistólica & & & $110,8 \pm 11,9$ \\
\hline Pressão arterial diastólica & & & $72,8 \pm 9,7$ \\
\hline Índice de massa corpórea $\left(\mathrm{kg} / \mathrm{m}^{2}\right)$ & & & $27,4 \pm 4,6$ \\
\hline Saudável: $<25$ & 53 & 34,4 & \\
\hline Sobrepeso: 25 |-| 29,9 & 66 & 42,9 & \\
\hline Obesidade: $\geq 30$ & 35 & 22,7 & \\
\hline Circunferência abdominal $(\mathrm{cm})$ & & & $91,3 \pm 13,1$ \\
\hline Normal: masculino: $<94$ / feminino: $<80$ & 46 & 29,9 & \\
\hline Risco aumentado: masculino: $\geq 94$ / feminino: $\geq 80$ & 50 & 32,5 & \\
\hline Risco muito aumentado: masculino: $\geq 102 /$ feminino: $\geq 88$ & 58 & 37,7 & \\
\hline Colesterol total $(\mathrm{mg} / \mathrm{dL})$ & & & $188,8 \pm 37,1$ \\
\hline Ótimo: $<200$ & 99 & 64,3 & \\
\hline Limítrofe: 200 |-| 239 & 38 & 24,7 & \\
\hline Alto: $\geq 240$ & 17 & 11,0 & \\
\hline LDC-c: Low Density Lipoprotein (mg/dL) & & & $116,3 \pm 30,6$ \\
\hline Ótimo: $<100$ & 52 & 33,8 & \\
\hline Desejável: 100 |—| 129 & 55 & 35,7 & \\
\hline Limítrofe: 130 |—I 159 & 35 & 22,7 & \\
\hline Alto: $160|-| 189$ & 10 & 6,5 & \\
\hline Muito alto: $\geq 190$ & 2 & 1,3 & \\
\hline HDL-c: High Density Lipoprotein (mg/dL) & & & $47,5 \pm 11,9$ \\
\hline Baixo: $<40$ & 43 & 27,9 & \\
\hline Limítrofe: 40 |— 60 & 94 & 61,0 & \\
\hline Alto: $>60$ & 17 & 11,0 & \\
\hline Triglicérides $(\mathrm{mg} / \mathrm{dL})$ & & & $128,1 \pm 85,2$ \\
\hline Ótimo: < 150 & 108 & 70,2 & \\
\hline Limítrofe: $150|-| 200$ & 21 & 13,6 & \\
\hline Alto: 201 |— 499 & 23 & 15,0 & \\
\hline Muito alto: $\geq 500$ & 2 & 1,2 & \\
\hline Glicemia (mg /dL) & & & $89 \pm 13,2$ \\
\hline Normal: $<110$ & 137 & 89,0 & \\
\hline Tolerância diminuída glicose: $>110$ e $<126$ & 13 & 8,4 & \\
\hline Diabetes mellitus: $\geq 126$ & 4 & 2,6 & \\
\hline Proteína C reativa $(\mathrm{mg} / \mathrm{dL})$ & & & $0,2 \pm 0,8$ \\
\hline Primeiro quartil: $<0,1$ & 41 & 26,6 & \\
\hline Segundo quartil: $0,1 ।-\mid 0,2$ & 51 & 33,1 & \\
\hline Terceiro quartil: $0,2-10,4$ & 38 & 24,7 & \\
\hline Quarto quartil: >0,5 & 24 & 15,6 & \\
\hline
\end{tabular}

Texto Contexto Enferm, Florianópolis, 2011 Jul-Set; 20(3): 435-44. 
Os resultados finais da análise multivariada dos fatores associados à presença de hipertensão arterial em decorrência de níveis alterados da pressão estão apresentados na tabela 3. Dessa forma, para a medida casual da pressão, o nível de HDL-c apareceu como um fator protetor. A chance de apresentar a pressão arterial alterada, ou seja na faixa de hipertensão, diminuiu em $25 \%$ nas pessoas que apresentaram HDL-c $>40 \mathrm{mg} / \mathrm{dL}$.

Para a MAPA, considerando a média do período de $24 \mathrm{~h}$, verificou-se que a chance de apresentar hipertensão aumentou em 2,71 vezes para as pessoas do sexo masculino e 9,98 vezes quando a glicemia estava acima de $110 \mathrm{mg} / \mathrm{dL}$. Já em relação às condições de trabalho, verificou-se que trabalhar cansado algumas vezes, raramente ou nunca diminui em 19,4\% e 17,4\%, respectivamente, a chance de apresentar pressão arterial alterada, em relação aos profissionais que referiram trabalham cansado frequentemente.
Para o período da vigília da MAPA, também verificou-se que ser do sexo masculino aumentou em três vezes a chances de hipertensão; a mudança de faixa da normalidade ( $>$ de $110 \mathrm{mg} / \mathrm{dL}$ ) aumenta-se em 18 vezes a chance de apresentar pressão arterial aumentada. Em relação ao índice de massa corporal a mudança na faixa de normalidade $\left(>25 \mathrm{Kg} / \mathrm{m}^{2}\right)$ elevou em $10 \%$ a chance de apresentar nível de pressão arterial compatível com hipertensão.

Na avaliação do período de sono da MAPA, a presença de hipertensão se associou com o índice de massa corporal e o fato de ter dormido durante o dia em que foi realizada a MAPA. Portanto, no índice de massa corporal a mudança da faixa de normalidade $\left(>25 \mathrm{Kg} / \mathrm{m}^{2}\right)$ a chance de apresentar aumento da pressão arterial aumenta em cerca de $12 \%$; a presença de sono diurno apareceu como um fator protetor diminuindo em $14,0 \%$ a chance de apresentar hipertensão.

Tabela 3 - Modelo de regressão logística múltipla: variáveis associadas à pressão arterial casual e MAPA alterada dos profissionais que no atendimento pré-hospitalar. São Paulo-SP, 2010

\begin{tabular}{|c|c|c|c|c|c|c|}
\hline Variável & $\begin{array}{l}\text { Parâmetro } \\
\text { estimado }\end{array}$ & $\begin{array}{l}\text { Erro } \\
\text { padrão }\end{array}$ & $\mathbf{p}$ & $\begin{array}{l}\text { Odds } \\
\text { Ratio }\end{array}$ & \multicolumn{2}{|c|}{ IC $95 \%$} \\
\hline \multicolumn{7}{|c|}{$\begin{array}{l}\text { Pressão arterial casual } \geq 140 / 90 \mathrm{mmHg} \text { e/ou } \\
\text { uso de anti-hipertensivos }\end{array}$} \\
\hline Intercepto & $-0,683$ & 0,177 & 0,00 & & & \\
\hline $\mathrm{HDL}-\mathrm{c}>40 \mathrm{mg} / \mathrm{dL}$ & $-1,360$ & 0,589 & 0,02 & 0,257 & 0,081 & 0,813 \\
\hline \multicolumn{7}{|l|}{ MAPA Período de 24 h >130/80 mmHg } \\
\hline Intercepto & $-0,902$ & 0,181 & 0,00 & & & \\
\hline Sexo masculino & 1,000 & 0,414 & 0,01 & 2,717 & 1,206 & 6,122 \\
\hline Trabalhar cansado algumas vezes & $-1,746$ & 0,642 & 0,00 & 0,174 & 0,050 & 0,614 \\
\hline Trabalhar cansado raramente/nunca & $-1,624$ & 0,599 & 0,00 & 0,197 & 0,061 & 0,638 \\
\hline Glicemia > $110 \mathrm{mg} / \mathrm{dL}$ & 2,301 & 0,947 & 0,01 & 9,983 & 1,560 & 63,881 \\
\hline \multicolumn{7}{|c|}{ MAPA Período de vigília > $135 / 85 \mathrm{mmHg}$} \\
\hline Intercepto & $-0,902$ & 0,181 & 0,00 & & & \\
\hline Sexo masculino & 1,177 & 0,435 & 0,00 & 3,245 & 1,385 & 7,606 \\
\hline Trabalhar cansado algumas vezes & $-1,813$ & 0,656 & 0,00 & 0,163 & 0,045 & 0,590 \\
\hline Trabalhar cansado raramente/nunca & $-1,954$ & 0,623 & 0,00 & 0,142 & 0,042 & 0,481 \\
\hline Glicemia > 110 mg /dL & 2,414 & 0,982 & 0,01 & 11,180 & 1,632 & 76,60 \\
\hline Índice de massa corporal > 25Kg/m² & 0,096 & 0,046 & 0,03 & 1,101 & 1,006 & 1,206 \\
\hline \multicolumn{7}{|c|}{ MAPA Período de sono $>120 / 70 \mathrm{mmHg}$} \\
\hline Intercepto & 0,539 & 0,168 & 0,00 & & & \\
\hline Índice de massa corporal > 25Kg/m² & 0,113 & 0,047 & 0,01 & 1,119 & 1,021 & 1,226 \\
\hline Presença de sono diurno & $-1,969$ & 0,391 & 0,00 & 0,140 & 0,065 & 0,300 \\
\hline
\end{tabular}

\section{DISCUSSÃO}

A prevalência de hipertensão arterial identificada pela medida casual da pressão arterial (33\%), foi semelhante aos achados de estudos de base populacional mais recentes em nosso meio, que indicam índices na faixa de 30\% a 33\%. ${ }^{12}$ Porém, quando comparados com dados de estudos em profissões específicas, verifica-se que o achado da presente investigação foi maior. Dessa forma, estudo nacional com trabalhadores da indústria de refrigerantes identificou prevalência de $27 \%{ }^{13}$; 
índices bem próximos foram encontrados em funcionários de um complexo hospitalar da cidade de São Paulo $(26 \%)^{10}$ e em trabalhadores de uma empresa metalúrgica e siderúrgica $(24,7 \%)^{14}$; e em profissionais de enfermagem de um Hospital Universitário da cidade de Cuiabá-MT, a prevalência foi de 20,8\%. ${ }^{15}$ Em achados internacionais os índices foram bem menos elevados, em paramédicos $9 \%{ }^{16}$ e em bombeiros americanos $17,7 \% .{ }^{17}$

Destaca-se que houve redução na prevalência de hipertensão quando considerou-se os valores da MAPA, principalmente a média do período de vigília $(26,6 \%)$, que é o melhor parâmetro de comparação com a medida casual, pois está isenta da influência do período do sono, contido na média das 24 horas da MAPA. Essa redução pode ser explicada pelo fenômeno do avental branco, como a hipertensão do avental branco, que se caracteriza por níveis pressóricos na medida casual compatíveis com hipertensão $(\geq 140 / 90 \mathrm{mmHg})$, porém, normais no período de vigília da MAPA $(<135 / 85 \mathrm{mmHg})$. No presente estudo, este fato esteve presente em $9,5 \%$ dos profissionais, excetuando-se aqueles que faziam uso de medicamentos anti-hipertensivos. A prevalência da hipertensão do avental branco é muito variada, com achados de $20 \%$ a $30 \%{ }^{4}$ e estudo realizado em nosso meio apontou índice de $20 \% .^{18}$ Há indicativos ainda, de que $70 \%$ dos indivíduos que apresentam esse comportamento pressórico serão hipertensos em um período de 10 anos. ${ }^{12}$

A prevalência da hipertensão arterial vem aumentado nas últimas décadas e no presente estudo, tal fato poderia estar relacionado à especificidade do atendimento pré-hospitalar. Os profissionais que atuam nesses serviços permanecem geralmente 24 horas de "prontidão", em condição permanente de estado de atenção e submetidos ao ruído constante de sirenes e acionamento das viaturas. Tal situação pode resultar em uma maior reação ao estado de alarme. ${ }^{19}$ Estudos demonstraram que os indivíduos com maior reação de alarme induzidos pelo estresse físico ou mental apresentam maiores níveis pressóricos. ${ }^{20,21}$

O estado permanente de alerta poderia também ter contribuído para a prevalência, em mais da metade dos profissionais (63\%), de níveis pressóricos compatíveis com hipertensão, no período de sono da MAPA. Tal achado também estaria associado à especificidade do trabalho no serviço pré-hospitalar, pois quando estão de plantão, o sono é frequentemente interrompido para $\mathrm{o}$ atendimento das ocorrências. $\mathrm{O}$ estresse físico e mental e a interrupção do sono contribuiriam para o maior nível pressórico avaliado pela MAPA. Mesmo que os participantes tenham relatado que dormiram durante o plantão, não houve queda suficiente da pressão arterial para que houvesse o descenso pressórico desejável nesse período. A privação de sono possui um impacto negativo sobre a saúde, resultando em aumentos dos níveis pressóricos e do níveis de cortisol; aumento dos hormônio grelina e diminuição da leptina; maior percepção de estresse e aumento do peso; além de que a privação crônica do sono está associada ao sobrepeso, obesidade, diabetes mellitus e elevação da pressão arterial. ${ }^{22}$ Acrescenta-se ainda, que ausência de queda da pressa arterial durante o sono está associada a lesão de órgãos alvo como presença de infartos lacunares, hipertrofia de ventrículo esquerdo e microalbuminúria. ${ }^{4}$

A presença do índice de massa corporal e glicemia alterados no modelo de regressão logística, como variáveis preditoras para hipertensão arterial, demonstra o impacto dessas variáveis nesse grupo de profissionais. Esses achados são similares ao do estudo de abrangência nacional baseado em dados do sistema de Vigilância de Fatores de Risco e Proteção para Doenças Crônicas por Inquérito Telefônico (VIGITEL), que verificou que a chance de hipertensão foi maior para os indivíduos com excesso de peso, diabetes, dislipidemia e de eventos cardiovasculares. ${ }^{23}$ Nessa mesma linha, estudo na cidade de São Paulo mostrou que nos hipertensos que referiram índice de massa corporal alterado houve associação com presença de diabete, hipercolesterolemia, pressão sistólica não controlada e uso de mais de um anti-hipertensivo. ${ }^{24}$ Em outra investigação, com 1265 mulheres idosas, também houve associação entre hipertensão e índice de massa corporal (Odds Ratio $=1,04) .{ }^{25}$ A obesidade é um problema mundial, principalmente pelo crescimento alarmante nas últimas décadas, e a ligação com a hipertensão é inegável. Adiciona-se a esse contexto, o alto grau de sedentarismo que também favorece o ganho do peso, e a elevada carga de trabalhado semanal. Uma jornada de trabalho exaustiva contribuiria para o estresse físico e mental, além de alteração no ritmo circadiano, ou seja do padrão sono/vigília. Essas pessoas possuem maior possibilidade de se tornarem obesas, por influência dos hormônios orexígeno, grelina, e anorexígeno, leptina, que aumentariam o tônus adrenérgico e consequentemente a pressão arterial. ${ }^{26}$ Outra possibilidade de alteração concomitante, seria que a elevação crônica do cortisol em indivíduos expostos ao 
estresse crônico causa uma hiperatividade do eixo hipotálamo-hipófise-adrenal promovendo a obesidade visceral e alteração no metabolismo da glicose. ${ }^{27}$ Por outro lado, destaca-se que nas pessoas estudadas o nível elevado da fração HDL colesterol foi fator de proteção, atuando no endotélio contra a agressão do processo inflamatório decorrente da elevação da pressão arterial.

Pertencer ao sexo masculino como variável preditora para hipertensão, corrobora dados de outros estudos. ${ }^{14,28}$ Considera-se que até os 50 anos o predomínio seja maior nos homens, com inversão a partir dessa faixa etária, muito em função das alterações hormonais nas mulheres, advindas da menopausa. ${ }^{12} \mathrm{~A}$ variável gênero, merece atenção especial no contexto da hipertensão arterial. Investigação ${ }^{29}$ sobre a prevalência da hipertensão arterial referida, percepção de sua origem e formas de controle em área metropolitana de São Paulo, mostrou que as mulheres foram as que mais procuraram pela assistência e pondera que as mulheres parecem ter uma percepção mais acurada de sua condição de saúde e também desenvolvem maiores relações com os serviços de saúde em razão de seus atributos e funções reprodutivas.

A relação entre trabalhar cansado mais frequentemente e hipertensão, foi outro achado que se destacou. O cansaço pode ser resultante da elevada carga horária de trabalho associado aos desgastes físicos e psicológicos. Segundo modelo demanda-controle ${ }^{30}$, os profissionais que atuam no atendimento pré-hospitalar teriam altas demandas psicológicas e baixo controle sobre as suas tarefas, expondo-os a situações psicológicas incontroláveis e dinâmicas. Muitas vezes essas pessoas atuam em um ambiente hostil e potencialmente perigoso, portanto, um trabalho com alto risco para desenvolver estresse mental e físico. O cansaço físico poderia resultar em maior risco, para o profissional e para a vítima devido às características do atendimento pré-hospitalar. O profissional necessita tomar decisões rápidas, baseadas quase sempre em seu julgamento clínico, que poderia estar comprometido pela presença do cansaço físico e mental. Porém, talvez como um mecanismo compensatório a presença de sono diurno foi um fator protetor para hipertensão e estudos têm verificado o efeito benéfico da sesta na pressão arterial. ${ }^{31,32}$

\section{CONSIDERAÇÕES FINAIS}

Os resultados do presente estudo ratificaram que a prevalência de hipertensão quando avaliada exclusivamente pela medida casual da pressão arte- rial pode apresentar índices mais elevados quando comparados com a MAPA, que afasta a influência do observador e do ambiente. Ressalta-se ainda, a importância das variáveis que correspondem a fatores de risco cardiovascular que se associaram à ocorrência da hipertensão arterial. A presença de estilos e hábitos de vida inadequados, aliados às características do trabalho da amostra estudada foram determinantes importantes para a presença de níveis da pressão arterial compatíveis com hipertensão arterial. Embora os estudos transversais não permitam atribuir casualidade às associações encontradas, o presente estudo teve por mérito avaliar um dos principais fatores de risco cardiovascular em um grupo específico de profissionais, em especial os resultantes do aumento do índice de massa corporal. Porém, o que mais chama a atenção é que se trata de um grupo de profissionais com formação na área da saúde. Muitas vezes, atuam em situações de atendimento às vítimas de complicações da hipertensão não controlada, como o acidente vascular encefálico e infarto agudo do miocárdio.

O grande desafio para todos, instituições e profissionais, é reconhecer e encontrar mecanismos para atuar nos fatores de risco modificáveis. É imperativo o estímulo à atividade física, a adoção de hábitos alimentares mais saudáveis para manutenção ou redução do peso corporal, eliminação do tabagismo e moderação na ingestão de bebida alcoólica. Dessa forma, pode-se minimizar a elevação dos níveis pressóricos, que podem surgir com o aumentar da idade e da influência genética. Como se trata de uma população bem esclarecida sobre o assunto, considera-se superada a fase de esclarecimentos, porém o processo de mudança de comportamento se faz necessário e principalmente acreditar que pode mudar. A adesão a diferentes práticas e estilos de vida é um processo complexo, que envolve fatores emocionais e barreiras concretas, de ordem prática e logística. Superar as exigências institucionais diante de uma jornada de trabalho exaustiva e muitas vezes com mais de um vínculo empregatício, não é tarefa fácil. Dessa forma, considera-se que os resultados do presente estudo possam ser úteis para que médicos e membros da equipe de enfermagem que compuseram a amostra, possam refletir sobre suas condições de saúde e viabilidade de uma proposta para amenizar ou eliminar os fatores de risco cardiovascular identificados.

\section{REFERENCIAS}

1. Ministério da Saúde (BR). Secretaria de Vigilância em Saúde. Secretaria de Gestão Estratégica e 
Participativa. Vigitel Brasil 2009: vigilância de fatores de risco e proteção para doenças crônicas por inquérito telefônico [online]. Brasília (DF): Ministério da Saúde; 2010 [acesso 2011 Abr 06]. Disponível em: http://189.28.128.100/dab/docs/ publicacoes/geral/publicacao_vigitel_2009.pdf

2. Pierdomenico SD, Cuccurullo F. Prognostic value of white-coat and masked hypertension diagnosed by ambulatory monitoring in initially untreated subjects: an updated meta analysis.Am J Hypertens. 2011 Jan; 24(1):52-8.

3. Puchades R, Ruiz-Nodar JM, Blanco F, Rodríguez F, Gabriel R, Suárez C. White-coat hypertension in the elderly. Echocardiographic analysis. A substudy of the EPICARDIAN project. Rev Esp Cardiol. 2010 Nov; 63(11):1377-81.

4. Alessi A, Brandão AA, Pierin A, Feitosa AM, Machado CA, Moraes Forjaz CL. IV Diretrizes para uso da monitorização ambulatorial da pressão arterial. Arq Bras Cardiol. 2005 Jul; 85 Suppl 2:1-18.

5. Aquino EMLL, Magalhães LBNC, Araujo MJ, Almeida MCC, Leto JP. Hipertensão arterial em trabalhadoras de enfermagem-padrão de ocorrência, diagnóstico e tratamento. Arq Bras Cardiol. 2001 Mar; 76(3):197-202.

6. Mion Junior D, Pierin AMG, Bambirra AP, Assunção $\mathrm{JH}$, Monteiro JM, Chinen RY. et al. Hypertension in employees of a university general hospital. Rev Hosp Clin Fac Med S Paulo. 2004 Dez; 59(6): 29-36.

7. Mattos CE, Mattos MA, Toledo DG, Siqueira AG. Avaliação da Pressão arterial em bombeiros militares filhos de hipertensos através da monitorização ambulatorial da pressão arterial. Arq Bras Cardiol. 2006 Dez; 87(6):741-6.

8. Stiefel P, Gimenez J, Miranda ML, Villar J, MuñizGrijalvo O, Pamies E, et al. Ambulatory blood pressure monitoring in physicians working in a hospital: is there in increase in the number of subjects with high workplace blood pressures? Int J Cardiol. 1994 Jul; 45(3):183-9.

9. Organização Mundial da Saúde. Obesidade: prevenindo e controlando a epidemia global. São Paulo: Roca; 2004.

10. Sociedade Brasileira de Cardiologia. III Diretrizes Brasileiras sobre Dislipidemias e Diretriz de Prevenção da Aterosclerose. Arq Bras Cardiol. 2001 Nov; 77(Supl III):1-48.

11. Sociedade Brasileira de Diabetes. Consenso Brasileiro sobre Diabetes 2008: diagnóstico e classificação do diabetes melito e tratamento do diabetes melito do tipo 2 [arquivo da internet]. Rio de Janiero (RJ):SBD; 2009 [acesso 2011 Abr 06]. Disponível em: http:// www.lojavirtualsbd.com.br/diretrizes/diretrizes2008-apenas-para-download.html

12. Sociedade Brasileira de Hipertensão. Sociedade Brasileira de Cardiologia. Sociedade Brasileira de Nefrologia. VI Diretrizes Brasileiras de
Hipertensão Arterial. Rev Bras Hipertensão. 2010 Jan-Mar;17(1):1-69.

13. Cassani RSL, Nobre F, Pazin Filho A, Schmidt A. Prevalência de fatores de risco cardiovascular em trabalhadores de uma indústria brasileira. Arq Bras Cardiol. 2009 Jul; 92(1):16-22.

14. Martinez MC, Latorre MRDO. Fatores de risco para hipertensão arterial e diabete melito em trabalhadores de empresa metalúrgica e siderúrgica. Arq Bras Cardiol. 2006 Out; 87(4):471-9.

15. Reiners AAO, Costa ALRC, Arruda ALG, Costa LMFC, Nogueira MS. Hipertensão arterial: perfil de saúde dos trabalhadores de enfermagem de um hospital universitário. Texto Contexto Enferm. 2004 Jan-Mar; 13:(2):241-9.

16. Kales SN, Tsismenakis AJ, Chunbai Z, Soteriades ES. Blood pressure in firefighters, police officers and other emergency responders. Am J Hypertension. 2009 Jan; 22(1):11-20.

17. Kales S, Soteriades E, Cristoudias S, Tucker S, Nicolau M, Cristiani D. Firefighter's blood pressure and employment status on hazardous materials teams in Massachusetts: a prospective study. J Occup Environ Med. 2002 Jul; 44:669-76.

18. Segre CA, Ueno RK, Warde KRJ, Accorsi TAD, Miname $\mathrm{MH}$, Chi CK et al. Efeito, hipertensão e normotensão do jaleco branco na Liga de Hipertensão do Hospital das Clínicas, FMUSP. Prevalência, características clínicas e demográficas. Arq Bras Cardiol. 2003 Mai; 80(2):117-21.

19. Kuorinka I, Korhonen O. Firefighters' reaction to alarm: ECG and heart rate study. J Occup Med. 1981 Nov; 23: 762-6.

20. Oliveira LB, Cunha AB, Martins WA, Abreu RFS, Barros LSN, Cunha DM, et al. Monitorização ambulatorial da pressão arterial e medida casual em hiper-reatores aos esforços. Arq Bras Cardiol. 2007 Mai; 88(5):565-72.

21. Gasperin D, Netuveli G, Dias-da-Costa JS, Pattussi MP. Efeito do estresse psicológico no aumento da pressão arterial: uma metanálise de estudos de coorte. Cad Saúde Pública. 2009 Abr; 25(4):715-26.

22. Knutson KL, Spiegel K, Penev P, Van Cauter E. The metabolic consequences of sleep deprivation. Sleep Med Rev. 2007 Jun; 11(3):163-78.

23. Ferreira SRG, Moura EC, Malta DC, Sarno F. Freqüência de hipertensão arterial e fatores associados: Brasil, 2006. Rev Saúde Pública. 2009 Nov; 43(Supl 2):98-106.

24. Mion Jr. D, Pierin AMG, Bensenor IB, Marin JCM, Costa KRA, Oliveira LF, et al. Hipertensão arterial na cidade de São Paulo: prevalência referida por contato telefônico. Arq Bras Cardiol. 2010; 95(1):99-106.

25. Oliveira SMJV, Santos JL, Lebrão ML, Duarte YAO, Pierin AMG. Hipertensão arterial referida em mulheres idosas: prevalência e fatores associados. Texto Contexto Enferm. 2008 Abr-Jun; 17:(2):241-9. 
26. Crispim CA, Zalcman I, Dáttilo M, Padilha HG, TufikS, Mello MT. Relação entre sono e obesidade: uma revisão da literatura. Arq Bras Endocrinol Metab. 2007 Out; 51(7):1041-9.

27. Björntorp P. Do stress reactions cause abdominal obesity and comorbidities? Obes Rev. 2001 May; 2(2):73-86.

28. Cesarino CB, Cipullo JP, Martin JFV, Ciorlia LA, Godoy MRP, Cordeiro JA, et al. Prevalência e fatores sociodemográficos em hipertensos de São José do Rio Preto. Arq Bras Cardiol. 2008 Jul; 91(1):31-5.

29. Sousa ALL. Educando a pessoa hipertensa. In: Pierin AMG. Hipertensão arterial - uma proposta para o cuidar. Barueri (SP): Manole; 2004. p.165-74.
30. Karasek RA, Theorell TG, Schwartz J, Pieper C, Alfredson L. Job, psychological factors and coronary heart disease. Swedish prospective findings and US prevalence findings using a new occupational inference method. Adv Cardiol. 1982 May; 29:62-7.

31. Gangwisch JE, Heymsfield SB, Boden-Albala B, Buijs RM, Kreier F, Pickering TG, et al. Short sleep duration as a risk factor for hypertension: analyses of the first national health and nutrition examination survey. Hypertension. 2006 May; 47(5):833-9.

32. Gomes MAM, Pierin AMG, Mion Jr D. The effect of siesta in parameters of cardiac structure and in interpretation of ambulatory arterial blood pressure monitoring. Arq Bras Cardiol. 2000 Abr; 74(4):314-18. 